\title{
The Choice of Interventional Treatment of Gastric Variceal Hemorrhage: What Is Better?
}

\author{
Moon Young Kim \\ Department of Internal Medicine and Cell Therapy and Tissue Engineering Center, Yonsei University Wonju College of Medicine, Wonju, Korea
}

See "Balloon-Occluded Retrograde Transvenous Obliteration versus Transjugular Intrahepatic Portosystemic Shunt for the Management of Gastric Variceal Bleeding" by Geunwu Gimm, et al. on page 704, Vol. 12. No. 6, 2018

Gastric variceal hemorrhage (GVH) is rare compared with esophageal variceal hemorrhage, but bleeding easily leads to massive hemorrhage and rebleeding rate and mortality rate are higher. Various types of treatment of GVH have been developed due to the diversity of gastric varices (GV) location, size and collateral veins of the GV. However, it is difficult to select the appropriate treatment method because the situation of each patient is different and these treatments are not available at all medical institutions.

Data on prophylactic treatment of GV that have not been bleeding in the past are lacking, and preventive treatment for GV patients without bleeding has not been performed in general. The use of non-selective beta blocker (NSBB) has been reported to have little prophylactic effect in GV. In some studies, endoscopic vascular obturation (EVO) with cyanoacrylate in large and high risk GV has shown to reduce the risk of first bleeding and mortality compared to NSBB or non-treatment. ${ }^{1}$ However, due to the invasiveness of the EVO procedure and the associated complications risk, it is not widely applied in clinical practice.

Gastroesophageal varices type 1 (GOV1) is associated with EV, and endoscopic band ligation (EBL) is often used for bleeding and prophylactic treatment. However, GV is larger, deeper and the covering mucosal layer is thicker than EV, and the risk of exposure to stomach acid and food is also higher than that of EV. Therefore, although the active GVH control success rate of EBL is similar to that of EVO, but the rate of rebleeding and related mortality are higher than in EVO. Therefore, EVO is considered as the first choice for GOV1 hemorrhage rather than EBL. ${ }^{2}$

In gastric fundic varices, endoscopic approach can be used with diagnosis and hemostasis. EVO is the primary treatment for active bleeding. The initial hemostasis rate of EVO is better than $90 \%$, but the rate of rebleeding after the procedure is relatively high, which is over 30\%. ${ }^{3}$ In addition, EVO is invasive and may cause complications such as systemic embolization including pulmonary embolism, stroke, and multi-organ infarction, so some institutes tend to avoid EVO. Recently, transjugular intrahepatic portosystemic shunt (TIPS) and balloon-occluded retrograde transvenous obliteration (BRTO) have been used instead of EVO.

The studies of the therapeutic effect of TIPS on GVH were very limited and mostly small scales. In a non-randomized study comparing TIPS and EVO, both treatments showed an initial success rate of $90 \%$ or more. However, TIPS had more adverse effects such as hepatic encephalopathy and stent obstruction and higher treatment costs. ${ }^{4}$ However, TIPS was very effective as a salvage treatment for patients with GVH that failed initial treatment. ${ }^{5}$ In the past uncovered stents using, complications such as stent stenosis and obstruction occurred in more than half of the cases in one year, but the rate of occlusion was reduced to $8 \%$ in covered stent. ${ }^{6}$

BRTO is a treatment mainly applied in Asia and sclerosant is injected into GV through gastrorenal shunt. BRTO has a high success rate of 95\% or more in hemostasis of fundal GVH, and it is reported that elimination of varices or no more rebreeding in more than 97\% curred. ${ }^{7}$ However, EVs may recur or worsen in $20 \%$ to $41 \%$ of patients after the procedure.

The BRTO is not possible when the diameter of gastrorenal shunt is larger than that of the balloon, and it is inconvenient to hold the balloon for several hours or more until the varices

Correspondence to: Moon Young Kim

Department of Internal Medicine, Wonju Severance Christian Hospital, Yonsei University Wonju College of Medicine, 20 Ilsan-ro, Wonju 26426, Korea

Tel: +82-33-741-1229, Fax: +82-33-741-0951, E-mail: drkimmy@yonsei.ac.kr pISSN 1976-2283 eISSN 2005-1212 https://doi.org/10.5009/gnl18464

@ This is an Open Access article distributed under the terms of the Creative Commons Attribution Non-Commercial License (http://creativecommons.org/licenses/by-nc/4.0) which permits unrestricted non-commercial use, distribution, and reproduction in any medium, provided the original work is properly cited. 
are hardened after injecting the sclerosant. There is also a risk of systemic embolism with a sclerosant when the balloon ruptures during the procedure. To remedy this disadvantage, a newly designed treatment is plug-assisted retrograde transvenous obstruction (PARTO). PARTO can prevent shunt and embolization by using specially designed vascular plug instead of balloon and can induce hemostasis and varices obturation quickly and easily by using gelatinous precondition instead of sclerosant. A recent prospective multicenter study showed that the procedure success rate was $98.6 \%$ and there was no rebleeding or hepatic encephalopathy during follow-up. In addition, 40\% of patients showed improvement of liver function. In the future, PARTO can be expected to be main procedure to control GV with gastrorenal shunt. ${ }^{8}$

If so, what treatment should we prioritize for GV and GVH management? In particular, what is the next step if EVO is not feasible or fails? Previous reports comparing TIPS and BRTO in control of GVH were small scales and have provided little criterion for clinical choice. In this regard, Gimm et al.'s study ${ }^{9}$ in this issue of Gut and Liver provides clinically significant information. Although it is retrospective study, it showed that BRTO is safer and better in immediate and long term clinical outcome through the large size population study. Patients in the BRTO group showed strong trends of higher immediate bleeding control rates and lower cumulative rebleeding rates than those in the TIPS group. BRT0 group also showed significantly longer GVH-free survival and overall survival than the TIPS group. In addition, although BRTO showed some aggravation of ascites, it showed improvement of liver function after procedure. ${ }^{9}$ This suggests that BRTO may improve intrahepatic perfusion by blocking the collateral shunt flow, while TIPS could exacerbate decrease of intrahepatic perfusion by the iatrogenic intrahepatic shunt against the original hepatic physiology. Therefore, in acute or hemodynamically stable GVH with gastrorenal shunt, BRTO is superior to TIPS in terms of procedure success rate, rebleeding prevention rate, and complication incidence.

However, the limitations of studies for the treatment of GVH are that the patients studied are inhomogeneous. Because most of these studies include acute active bleeding, salvage treatment, secondary treatment after spontaneous hemostasis, or prevention of rebleeding at the same time, it is difficult to compare treatment effects according to each case. For clinical practice, it is necessary to propose a guideline through prospective comparative study between EVO and radiologic intervention treatments such as BRTO or PARTO for acute bleeding and secondary prophylaxis. In addition, EVO has an exaggerated worry about the risk of complications and in many cases, incomplete procedure with insufficient amount of cyanoacrylate injection is the main cause of rebleeding after EVO.

In summary, there are EVO, TIPS, BRTO, and PARTO for the treatment of GVH, and randomized studies comparing their effects are not enough. Therefore, currently in determining the first line treatment, it is necessary to comprehensively consider the feasibility of each institute, the severity of bleeding, and the patient status. Through previous studies, BRTO seems to have a better effect on early hemostasis, prevention of rebleeding and long-term outcome than TIPS. However, there is still no specific indication for which treatment to choose based on acute active bleeding, salvage treatment, secondary treatment after spontaneous hemostasis, or rebleeding prevention and well-designed prospective studies are needed in the future.

\section{CONFLICTS OF INTEREST}

No potential conflict of interest relevant to this article was reported.

\section{REFERENCES}

1. Mishra SR, Sharma BC, Kumar A, Sarin SK. Primary prophylaxis of gastric variceal bleeding comparing cyanoacrylate injection and beta-blockers: a randomized controlled trial. J Hepatol 2011;54:1161-1167.

2. Park SJ, Kim YK, Seo YS, et al. Cyanoacrylate injection versus band ligation for bleeding from cardiac varices along the lesser curvature of the stomach. Clin Mol Hepatol 2016;22:487-494.

3. Jun $\mathrm{CH}$, Kim KR, Yoon JH, et al. Clinical outcomes of gastric variceal obliteration using N-butyl-2-cyanoacrylate in patients with acute gastric variceal hemorrhage. Korean J Intern Med 2014;29:437-444.

4. Mahadeva S, Bellamy MC, Kessel D, Davies MH, Millson CE. Costeffectiveness of N-butyl-2-cyanoacrylate (histoacryl) glue injections versus transjugular intrahepatic portosystemic shunt in the management of acute gastric variceal bleeding. Am J Gastroenterol 2003;98:2688-2693.

5. Barange K, Péron JM, Imani K, et al. Transjugular intrahepatic portosystemic shunt in the treatment of refractory bleeding from ruptured gastric varices. Hepatology 1999;30:1139-1143.

6. Barrio J, Ripoll C, Bañares R, et al. Comparison of transjugular intrahepatic portosystemic shunt dysfunction in PTFE-covered stentgrafts versus bare stents. Eur J Radiol 2005;55:120-124.

7. Park JK, Saab S, Kee ST, et al. Balloon-occluded retrograde transvenous obliteration (BRTO) for treatment of gastric varices: review and meta-analysis. Dig Dis Sci 2015;60:1543-1553.

8. Gwon DI, Kim YH, Ko GY, et al. Vascular plug-assisted retrograde transvenous obliteration for the treatment of gastric varices and hepatic encephalopathy: a prospective multicenter study. J Vasc Interv Radiol 2015;26:1589-1595.

9. Gimm G, Chang Y, Kim HC, et al. Balloon-occluded retrograde transvenous obliteration versus transjugular intrahepatic portosystemic shunt for the management of gastric variceal bleeding. Gut Liver 2018;12:704-713. 